\title{
Was ist „gutes Sterben“?
}

\section{Begriffsklärung und Stand der Forschung}

Nico Hutter, Ulrich Stößel, Cornelia Meffert, Mirjam Körner, Claudia Bozzaro, Gerhild Becker, Harald Baumeister

\begin{abstract}
Die Fortschritte der modernen Medizin heben nicht nur die durchschnittliche Lebenserwartung an - sie verlagern auch das Sterben vom privaten in den öffentlichen Raum. In den westlichen Industrienationen sterben heute die meisten Menschen im fortgeschrittenen Alter und in medizinischen Einrichtungen. So stellt sich die Frage, wie die Medizin ein „gutes Sterben“ ermöglichen kann. Was aber verstehen Patienten, Angehörige und Versorger eigentlich unter einem „guten Sterben“? Dieser Frage geht der folgende Artikel nach.
\end{abstract}

Todesfälle in Deutschland | Im Jahr 2010 verstarben in Deutschland 858768 Menschen [32]. Die mit Abstand häufigsten Todesursachen waren dabei Herz-Kreislauf-Erkrankungen $(41,1 \%)$ sowie Krebs (25,5\%) [32]. Weltweit sind lebensbedrohliche Erkrankungen wie Krebs und HIV eine große Belastung [31]. Die Fortschritte in der Medizin haben zu einer Verlängerung der Lebenszeit geführt. Ein immer größerer Teil der Bevölkerung wird älter und pflegebedürftig, der Bedarf an unterstützenden und palliativen Versorgungsangeboten steigt [5].

Versorgungsdefizite I Der medizinische Fortschritt hat jedoch auch negative Folgen: Bereits die SUPPORT-Studie aus den USA zeigte, dass für manche Patienten der Sterbeprozess (unnötig) verlängert wird und dass die Kommunikation zwischen Ärzten, Patienten und Familien verbesserungsbedürftig ist [37]. Neuere Studien aus Europa zeigen ebenfalls Defizite in der Versorgung sterbenskranker Patienten auf $[7,11]$. So findet bei sterbenden Patienten häufig keine Palliativversorgung im Akutkrankenhaus statt, und nur in einem Viertel der Sterbefälle sind Angehörige während des Sterbens anwesend [11]. Darüberhinaus schätzen lediglich ein Drittel der Pflegekräfte im Krankenhaus die Qualität des Sterbens als zufriedenstellend ein [11]. Die 2008/2009 in Deutschland durchgeführte EPACS-Studie [27] konstatierte aufgrund erster Ergebnisse, dass eine deutliche Lücke zwischen dem Bedarf an spezialisierter Versorgung und tatsächlicher Nutzung solcher Angebote besteht.

Umfragen zum Thema | Auch die Medien griffen das Thema „gutes Sterben“ in jüngerer Berichterstattung auf $[12,39]$. Eine Spiegel-Umfrage ergab, dass zwei Drittel der Deutschen glauben, die Gesellschaft verdränge das Thema Tod [39]. Des Weiteren hoffen 67 Prozent der Befragten auf ein schnelles und plötzliches Sterben aus einer guten gesundheitlichen Verfassung heraus [39]. Ähnliche Ergebnisse zeigen sich in einer aktuellen
Befragung des Deutschen Hospiz- und Palliativverband e.V. aus dem Jahr 2012. Hier gab die Mehrzahl der telefonisch Befragten (58\%) an, dass sich die Gesellschaft mit dem Thema Sterben und Tod zu wenig befasse [9]. Lediglich 54\% der Befragten gaben an, sich ab und zu oder häufig über das eigene Sterben Gedanken zu machen [9]. Vor diesem Hintergrund stellt sich die Frage, was aus persönlicher wie auch aus gesellschaftlicher Sicht ein „gutes Sterben“ ist.

Die vorhandene Literatur zeigt, dass es Defizite in der Versorgung sterbenskranker Patienten gibt. Gleichzeitig wird das Thema Sterben von einer Mehrheit kaum thematisiert.

\section{Stand der Forschung: Definition von „gutem Sterben“}

Nach Göckenjan besteht in der Öffentlichkeit Konsens, dass das Sterben „human, würdig und gut sein soll“ $[13$, S.8]. Es gibt jedoch in der vorhandenen Literatur keine einheitliche Definition des Konzepts „gutes Sterben“. Vielmehr finden sich verschiedene synonyme Begriffe für „gutes Sterben“ wie „angemessenes Sterben“, „gesundes Sterben“, „richtiges Sterben“, „glückliches Sterben“ oder „friedliches Sterben“. Mak und Clinton [19] zeigen, dass der Begriff des „guten Sterbens“ am häufigsten verwendet wird. Eine Annäherung an das, was unter dem Begriff des „guten Sterbens" verstanden wird, ist durch eine Beschreibung der Attribute, die aus Sicht von Patienten und Versorgern ein gutes Sterben auszeichnen, zu erlangen [14].

Ähnlich wie das Konstrukt „Lebensqualität“ hat sich in der Literatur das Konzept der „Quality of death and dying“ etabliert [25]. Hales und Kollegen [15] differenzieren hier 3 verschiedene Ebenen, auf denen einzelne Attribute des „guten Sterben“ unterschieden werden können: 
1. Die Lebensqualität am Ende des Lebens, welche multidimensional Aspekte des körperlichen, psychischen und spirituellen/ seelischen Wohlbefindens umfasst

2. Die Qualität des Sterbeprozesses, die die Dimensionen der Lebensqualität am Ende des Lebens umfasst und diese um spezifische Aspekte, die mit dem Sterbeprozess zusammenhängen, erweitert

3. Die Qualität der Versorgung am Ende des Lebens, die sich darauf bezieht, inwieweit die Versorgung auf die Bedürfnisse Sterbender und ihrer Angehörigen ausgerichtet ist und die Rahmenbedingungen für ein patientenorientiertes und autonomes Sterben bereitstellt.

Des Weiteren ist „gutes Sterben“ mit verschiedenen psychosozialen Korrelaten assoziiert, und es können Determinanten benannt werden, die auf Versorgungsebene für „gutes Sterben“ unabdingbar sind.

Ähnlich dem Konstrukt der Lebensqualität kann von der Qualität des Sterbens gesprochen werden. Eine Annäherung ist möglich durch eine Beschreibung der damit verbundenen Attribute aus Sicht von Patienten und Versorgern.

\section{Attribute „guten Sterbens“}

Neuere Studien versuchten, aus verschiedenen Perspektiven „gutes Sterben“ zu erfassen. Meist werden dabei beschreibende Attribute und Präferenzen aus Sicht von Patienten, Angehörigen und Gesundheitsberufen erfasst $[14,19]$, ( Tab. 1).

Lebensqualität am Ende des Lebens | Mit Blick auf diese Ebene nennen Patienten, Angehörige, Ärzte und Pflegekräfte die Linderung von Schmerzen und die Behandlung von Krankheitssymptomen als zentrales Attribut für „gutes Sterben“ [14, 19, 21, 26, 33, 34] ( Tab. 1). In einer Bevölkerungsbefragung in den Niederlanden bei 1777 erwachsenen Personen gaben $87 \%$ der Befragten an, schmerzfrei sterben zu wollen [30].

- Darüber hinaus wichtige Attribute sind das psychische Wohlbefinden, die körperliche sowie kognitive Funktionsfähigkeit [21], die Qualität der sozialen Beziehungen (v.a. zu den engen Familienangehörigen) $[14,21,34]$ und die Aufrechterhaltung von Hoffnung und Freude [19, 21] - aus Sicht von Patienten und Angehörigen.

Aus Sicht von Ärzten und Pflegekräften steht in Bezug auf die Lebensqualität am Ende des Lebens die Schmerzlinderung sowie die Antizipation und das Management von Krankheitssymptomen im Vordergrund $[14,19]$.
Qualität des Sterbens I Mit Blick auf diese Ebene nennen Ärzte wie Pflegekräfte Attribute „guten Sterbens“, die weitestgehend auch aus Sicht von Patienten und Angehörigen bedeutsam sind.

- Zuerst ist der Wunsch zu nennen, einen hinausgezögerten Tod zu vermeiden [14]. In einer qualitativen Studie bei 35 AIDS-Patienten wünschten sich einige Befragte auch die Möglichkeit zum assistierten Suizid, um unerträgliche Schmerzen zu vermeiden [26].

- Des Weiteren geben Patienten und Angehörige an, ein Gefühl von Kontrolle bewahren zu wollen [19]. So finden es $88 \%$ der niederländischen Allgemeinbevölkerung wichtig, am Ende des Lebens in der Lage zu sein, Entscheidungen im Hinblick auf Pflege und Versorgung treffen zu können [30].

AIDS-Patienten geben an, an den Behandlungsentscheidungen am Ende des Lebens beteiligt sein zu wollen. Das beinhaltet auch die Entscheidung über das Ende der Behandlung [26]. Des Weiteren berichten Menschen aus der japanischen Allgemeinbevölkerung, an einem von ihnen bestimmten und bevorzugten Ort sterben zu wollen [21].

Nach Mak und Clinton [19] ergeben sich je nach Sterbeort verschiedene Anforderungen. Im Hospiz bestehen professionelle Rahmenbedingungen für einen „guten Tod“ - jedoch ist man hier mit einer fremden Umgebung und dem Sterben anderer konfrontiert [19]. Die meisten Menschen wollen zwar zuhause im familiären Umfeld sterben [3, 26], jedoch wollen viele nicht zur Belastung für andere werden [21, 33].

Der Anwesenheit nahestehender Personen wird überwiegend große Bedeutung beigemessen [14, 21, 26, 33]. Die Vollendung des Lebens in hohem Alter und die Beendigung wichtiger Aufgaben im Leben wird von vielen Patienten als Voraussetzung für „gutes Sterben“ erachtet [19, 21, 26, 33, 34]. Dazu gehören die Erfüllung persönlicher Wünsche, die Lösung bestehender persönlicher Konflikte und das Abschiednehmen von den Angehörigen [19, 21, 26, 33, 34, 30]. Im Sterbeprozess ist die Akzeptanz und die Bewusstheit des eigenen Sterbens sowie das Vorbereitetsein auf den Tod für die Patienten bedeutsam, um dem Leben Sinn und Bedeutung zu geben [19, 34]. In diesem Zusammenhang geben 92\% der Befragten aus der niederländischen Allgemeinbevölkerung an, mit Würde sterben zu wollen [30]. Auch die Erfüllung von spirituellen/religiösen Bedürfnissen wird von Patienten und Angehörigen häufig genannt [26, 33].

Qualität der Versorgung am Lebensende I Eine qualitative Studie mit Patienten auf Intensivstationen zeigt, dass sich Patienten und Angehörige eine klare und mitfühlende Kommunikation 


\begin{tabular}{|c|c|c|}
\hline Ebene & Perspektive & Attribute \\
\hline \multirow[t]{2}{*}{$\begin{array}{l}\text { Lebensqualität am } \\
\text { Ende des Lebens }\end{array}$} & Patienten und Angehörige & $\begin{array}{l}\text { Linderung von Schmerzen und anderen Symptomen } \\
\text { Psychisches Wohlbefinden } \\
\text { Soziale Beziehungen } \\
\text { Hoffnung und Freude aufrechterhalten }\end{array}$ \\
\hline & Versorger & $\begin{array}{l}\text { Schmerzlinderung } \\
\text { Antizipation und Management von Krankheitssymptomen }\end{array}$ \\
\hline \multirow[t]{2}{*}{$\begin{array}{l}\text { Qualität des } \\
\text { Sterbeprozesses }\end{array}$} & Patienten und Angehörige & $\begin{array}{l}\text { Verlängertes Sterben vermeiden (zum Teil auch Wunsch nach assistiertem } \\
\text { Suizid) } \\
\text { Ein Gefühl von Kontrolle / Autonomie bewahren } \\
\text { An einem bevorzugten Ort zu sterben } \\
\text { Anwesenheit der nahestehenden Angehörigen zum Zeitpunkt des Sterbens } \\
\text { Nicht zur Belastung für andere zu werden } \\
\text { Vollendung / Abschluss (unerledigte Dinge und Probleme lösen, Abschied } \\
\text { nehmen, mit sich und anderen Frieden schließen) } \\
\text { Akzeptanz des Sterbens und Vorbereitetsein } \\
\text { Sterbeprozess (bei Bewusstsein oder im Schlaf) } \\
\text { Spirituelle (religiöse) Aspekte des Sterbens }\end{array}$ \\
\hline & Versorger & $\begin{array}{l}\text { Anwesenheit der nahestehenden Angehörigen zum Zeitpunkt des Sterbens } \\
\text { Bewusstheit und Vorbereitetsein des Patienten } \\
\text { Würde und Autonomie des Patienten bewahren } \\
\text { Private und friedliche Atmosphäre schaffen } \\
\text { Akzeptanz des Sterbens }\end{array}$ \\
\hline \multirow[t]{2}{*}{$\begin{array}{l}\text { Versorgungsqualität } \\
\text { am Ende des Lebens }\end{array}$} & Patienten und Angehörige & $\begin{array}{l}\text { Gute Kommunikation durch Ärzte und Versorger } \\
\text { Patientenorientierung } \\
\text { Klinische Versorgung, die auf Verringerung des Leidens ausgerichtet ist unc } \\
\text { Würde, Persönlichkeit und Privatsphäre der Patienten wahrt } \\
\text { Anwesenheit der Familie ermöglichen und emotionale Unterstützung für } \\
\text { Familie } \\
\text { Gutes Verhältnis zu Ärzten und Pflegern } \\
\text { Gute Versorgungsqualität }\end{array}$ \\
\hline & Versorger & $\begin{array}{l}\text { Optimales Management der Symptome und umfassende Betreuung von } \\
\text { Patienten und Angehörigen } \\
\text { Vorhandensein von Leitlinien zur Versorgung Sterbender } \\
\text { Mehr Pflegekräfte } \\
\text { Vorbereitung auf das Thema Tod in der Ausbildung } \\
\text { Vorhandensein einer Vorsorgevollmacht oder einer Patientenverfügung }\end{array}$ \\
\hline
\end{tabular}

Tab. 1 Attribute "guten Sterbens" eingeteilt nach Ebene und Perspektive durch Ärzte und Pfleger und die Berücksichtigung ihrer Präferenzen bei der Behandlung wünschen [24]. Eine weitere qualitative Studie zeigte ähnliche Befunde, gute Kommunikation und Beteiligung an der Entscheidungsfindung wurde von den Patienten als stärkend empfunden [34].

- Die Behandlung soll auf die Verringerung des Leidens ausgerichtet sein und Würde, Persönlichkeit und Privatsphäre der Patienten wahren [24].

- Die klinische Versorgung soll die Nähe der Angehörigen zum Sterbenden ermöglichen und emotionale Unterstützung für die Angehörigen anbieten [24].

- Patienten und Angehörige nennen zudem ein vertrauensvolles und empathisches Verhältnis zwischen Ärzten und Pflegern auf der einen Seite und Patienten auf der anderen Seite [21, $26,33,34$ ] sowie eine gute Versorgungsqualität im Allgemeinen [26].
Die Versorger (Ärzte, Pflegekräfte) nennen zur Ebene der "Qualität der Versorgung am Lebensende" vorwiegend Attribute für ein "gutes Sterben“, die sich auf die Versorgungsstruktur und ihre Prozesse beziehen.

Neben dem optimalen Management der Symptome und einer umfassenden Betreuung von Patienten und Angehörigen [14] nennen Pflegekräfte

- Leitlinien zur Versorgung sterbender Patienten, - eine höhere Quote an Pflegekräften pro Patient und

- das Vorhandensein von Vorsorgevollmacht bzw. Patientenverfügung

als wichtige Attribute für ein „gutes Sterben“ [11]. Auch die persönliche Vorbereitung auf das Thema Tod wird in den palliativmedizinischen Berufsgruppen als wichtiges Attribut genannt 
[34]. Darüber hinaus sollte das Gesundheitssystem sterbenden Menschen ein Versorgungsangebot machen, das von professionell Behandelnden umgesetzt wird und auch räumlich eine optimale Versorgung ermöglicht [35]

Nach Stewart et al. [35] sind medizinische Entscheidungsprozesse, die die Patientenwünsche berücksichtigen, adäquate Informationsvermittlung und Betreuung von Patient und Familie sowie interpersonelle und kommunikative Fähigkeiten unabdingbare Faktoren für ein „gutes Sterben“ [35].

\section{Korrelate „guten Sterbens“}

Neben den beschreibenden Attributen identifizieren vorhandene Studien Variablen, die mit „gutem Sterben“ assoziiert sind (Korrelate).

- Patientenfaktoren, die mit „gutem Sterben“ zusammenhängen, sind Diagnose, Schweregrad und Verlauf der Erkrankung, die finanzielle Situation der Patienten, kulturelle Wertvorstellungen und religiöser Glauben sowie das Alter [14].

In einer Befragung der niederländischen Allgemeinbevölkerung wurden Patientencharakteristika untersucht, die mit bestimmten Präferenzen hinsichtlich des Sterbens assoziiert sind [30].

- So findet sich dort, dass Geschlecht (weiblich), Religiosität und ein Leben in Partnerschaft mit dem Wunsch, zuhause zu sterben, zusammenhängen.

- Den Moment des Sterbens bestimmen zu wollen, ist für Menschen ohne religiösen Glauben, mit geringerer Schuldbildung und ohne Lebenspartner von Bedeutung.

- Ältere Menschen äußerten häufiger die Sorge, von anderen abhängig zu sein und zur Belastung zu werden, während jüngere Teilnehmer und solche mit geringerer Schulbildung häufiger den Wunsch äußerten, bis zum Tod bei Bewusstsein zu sein. Teilnehmer mit religiösem Glauben hofften häufiger, existenzielle Fragen geklärt zu haben und sich auf den Tod vorbereiten zu können [30].

In einer Befragung der japanischen Allgemeinbevölkerung zeigt sich ein Zusammenhang zwischen Alter und Geschlecht mit der subjektiven Wichtigkeit einzelner Attribute „guten Sterbens“ [21]. So wünschten sich sowohl ältere Teilnehmer aus der Allgemeinbevölkerung als auch ältere Teilnehmer aus Familien, die einen Angehörigen verloren haben, signifikant häufiger, sanft und nicht bei Bewusstsein zu sterben. Darüberhinaus maßen ältere Befragte aus trauernden Familien religiösen und spirituellen Aspekten sowie körperlicher und kognitiver
Funktionsfähigkeit mehr Bedeutung bei als jüngere Teilnehmer aus Familien, die einen Angehörigen verloren haben. In dieser Studie war das Geschlecht (weiblich) mit dem Wunsch nach einer guten Beziehung zu Ärzten und Pflegekräften assoziiert [21].

Patientenfaktoren wie Diagnose, Schweregrad, Krankheitsverlauf, finanzielle Situation, kulturelle Wertvorstellungen, Religiosität und Alter werden in der Literatur als Korrelate „guten Sterbens“ identifiziert.

\section{Gesellschaftliche Aspekte "guten Sterbens“}

Patientenorientierung I Die beschriebenen Befunde zeigen, dass eine gesellschaftliche Diskussion über das Sterben notwendig ist. Es deutet sich ein Konsens an, bei dem eine Patientenorientierung im Vordergrund stehen sollte [13]. Dabei ist die Frage wichtig, welche Bedingungen die Gesellschaft herstellen kann, um „gutes Sterben“ zu ermöglichen. Wie soll die Versorgung ausgerichtet werden, wenn es nach den Bedürfnissen von Patienten und Angehörigen sowie den im Versorgungssystem tätigen Berufsgruppen geht? Die beschriebenen Attribute „guten Sterbens“ können dafür als Diskussionsrahmen dienen.

Im (palliativ)medizinischen Kontext wie in der breiten Öffentlichkeit werden aktuell Themen wie

- Patientenverfügungen,

- palliative Sedierung und

- ärztlich assistierter Suizid

breit und intensiv diskutiert. Dabei wird der Selbstbestimmung von Patienten große Bedeutung beigemessen.

Patientenverfügungen | In Patientenverfügungen äußern Patienten schriftlich ihre Therapiewünsche und lehnen bestimmte Maßnahmen ab - für den Fall, dass sie sich in einer späteren klinischen Situation nicht mehr selbst äußern können [3]. In einer aktuellen Befragung des Deutschen Hospizund Palliativverbands in der deutschen Allgemeinbevölkerung gaben $91 \%$ der Befragten an, das Thema Patientenverfügung zu kennen [9]. Insgesamt haben 26\% der Befragten (42\% der Befragten über 60 Jahre) eine Patientenverfügung verfasst und $43 \%$ schon einmal ernsthaft darüber nachgedacht [9]. Bei der Subgruppe derer, die keine Patientenverfügung erstellt haben, geben $43 \%$ an, das Thema verdrängt bzw. verschoben zu haben [9].

- Diese Verdrängung zeugt evtl. von der Schwierigkeit, die sich beim Formulieren einer Patientenverfügung ergibt. Es ist nicht immer 


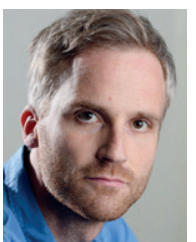

Dr. Nico Hutter

ist Wissenschaftler in der Medizinischen Psychologie und Medizinischen

Soziologie der Universität Freiburg

nicohutter@web.de

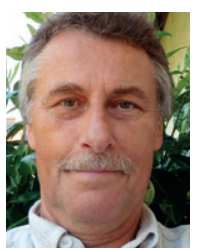

Dr. Ulrich Stößel

ist Lehrbeauftragter der Medizinischen Psychologie und Medizinischen

Soziologie der Universität Freiburg und Geschäftsführer der Freiburger Forschungsstelle Arbeitsund Sozialmedizin GbR ulrich.stoessel@mps. uni-freiburg.de

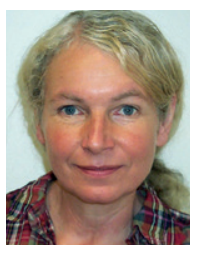

Dr. Cornelia Meffert ist Senior-Researcher an der Klinik für Palliativmedizin des Universitätsklinikums Freiburg cornelia.meffert@uniklinikfreiburg.de

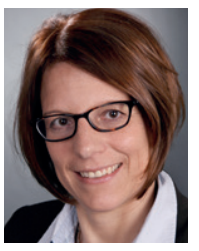

PD Dr. Mirjam Körner ist wissenschaftliche Mitarbeiterin in der Medizinischen Psychologie und Medizinischen Soziologie der Universität Freiburg mirjam.koerner@mps. uni-freiburg.de leicht, sich in zukünftige Krankheitsszenarien hineinzudenken und zu antizipieren, welche Therapiemaßnahmen man sich dann vermutlich wünschen oder ablehnen würde [18].

- So kann z.B. die festgehaltene Ablehnung intensivmedizinischer Maßnahmen dazu führen, dass auch eine temporär notwenige Maßnahme unter Umständen mit fatalen Folgen nicht eingesetzt werden könnte. Daher ist nach Maio [18] ein autonomes und geplantes Sterben nicht immer automatisch auch ein „gutes Sterben“.

Patientenverfügungen dokumentieren den Patientenwillen in Bezug auf bestimmte Behandlungsmaßnahmen. Sie gelten für den Fall, dass ein Patient sich in einer bestimmten klinischen Situation nicht mehr selbst äußern kann. Jedoch lassen sich nicht alle Eventualitäten eines Krankheitsverlaufes und der vorhandenen Behandlungsmöglichkeiten vorhersehen.

Palliative Sedierung I Die palliative Sedierung wird zur Symptomlinderung in der Palliativmedizin eingesetzt. Sie soll schwerstes Leiden verhindern und ein friedliches Sterben ermöglichen. Durch eine medikamentöse Dämpfung des Bewusstseins werden die Patienten dabei in einen narkoseähnlichen Zustand versetzt [3].

- 94\% der Palliativmediziner befürwortet die Praxis der palliativen Sedierung [23].

- In einer aktuellen europäischen Studie wird die Häufigkeit der Anwendung palliativer Sedierungsmaßnahmen für Holland mit $11 \%$, Belgien mit 20\% und Großbritannien mit 17\% angegeben [1].

Darüberhinaus zeigten sich in einer weiteren vergleichenden Studie zwischen 6 europäischen Ländern Raten zwischen 2,5\% (Dänemark) und $8,5 \%$ (Italien) [23]. Dabei ist besonders interessant, welche Faktoren die Anwendung palliativer Sedierungsmaßnahmen beeinflussen. So war die Wahrscheinlichkeit für eine kontinuierliche und tiefe Sedierung bis zum Eintritt des Todes für männliche und junge Patienten sowie Patienten mit bösartigen Erkrankungen erhöht [1]. Auch ist die palliative Sedierung im Krankenhaus signifikant häufiger als an anderen Sterbeorten [1].

- Der Wunsch, das Sterben nicht bei Bewusstsein erleben zu müssen, ist in der Allgemeinbevölkerung weit verbreitet. So geben lediglich $14 \%$ der Frauen und 12\% der Männer der deutschen Allgemeinbevölkerung an, bewusst und begleitet sterben zu wollen [8].

- Im Gegensatz dazu geben jedoch 92\% der Patienten mit fortgeschrittenen schweren Erkrankungen an, dass sie es für wichtig halten, im Sterbeverlauf bei klarem Bewusstsein zu sein [33].
Diese Diskrepanz spiegelt evtl. die Veränderung der Einstellungen zu Tod und Sterben im Verlauf der Sterbephase wider - sie sollte bei der Indikationsstellung zur palliativen Sedierung berücksichtigt werden. Bis jetzt gibt es keine evidenzbasierten und validierten Methoden, die das optimale Timing für Beginn, Abbruch und Auswahl von palliativmedizinischen Maßnahmen erlauben [28].

Ärztlich assistierter Suizid | In Zusammenhang mit der steigenden Lebenserwartung und der hohen Prävalenz chronischer Erkrankungen steht die Diskussion um den freiverantwortlichen und ärztlich assistierten Suizid [16]. Diese Diskussion konzentriert sich auf unheilbar kranke Patienten [3], bei denen der Wunsch, den Zeitpunkt des eigenen Todes zu bestimmen, besonders bedeutsam ist.

- Beim ärztlich assistierten Suizid tötet sich der Betroffene selbst (z.B. durch eine tödliche Medikamentendosis), wird dabei jedoch durch Dritte unterstützt - z.B. durch einen Arzt, der das Medikament zur Selbsttötung verschreibt [3].Eine in 23 europäischen Ländern durchgeführte Trendanalyse (1981-2008) zur Akzeptanz der ärztlich assistierten Sterbehilfe zeigt für westeuropäische Länder eine signifikante Zunahme der Akzeptanz in der Bevölkerung. Dies ist in den osteuropäischen Ländern, oft auch aufgrund stärkerer religiöser Bindungen, nicht in der gleichen Form zu beobachten [4].

- Im Gegensatz zur vorherrschenden Meinung in der Allgemeinbevölkerung sprechen sich rund 75\% der Palliativmediziner gegen eine Legalisierung des ärztlich assistierten Suizids und über $90 \%$ gegen eine Legalisierung der aktiven Euthanasie aus [23].

- Nach Dahl [6] lässt sich das zum Teil damit erklären, dass gleichzeitig $94 \%$ der befragten Palliativmediziner die Anwendung palliativer Sedierungsmaßnahmen befürworten und daher möglicherweise keinen Bedarf für eine gesetzliche Zulassung des ärztlich assistierten Suizids oder der aktiven Euthanasie sehen.

Die Ergebnisse einer weiteren Studie deuten in eine ähnliche Richtung. In einer Befragung von 988 Patienten im Endstadium ihrer Erkrankung befürworteten zwar 60,2\% der befragten Patienten das Recht zum ärztlich assistierten Suizid, jedoch gaben lediglich 10,6\% der Befragten an, ernsthaft darüber nachzudenken, ärztlich assistierten Suizid für sich in Anspruch nehmen zu wollen [10].

Im Gegensatz zur teils mehrheitlichen Zustimmung in der Allgemeinbevölkerung sprechen sich rund $75 \%$ der Palliativmediziner gegen eine Legalisierung des ärztlich-assistierten Suizids aus. 
Konsequenz für Klinik und Praxis

- Um die gesellschaftliche Diskussion über das Wesen des „guten Sterbens“ zu vertiefen, braucht es weitere Forschung. Künftige Studien müssen klären, welche Bedeutung und Gewichtung die oben genannten Attribute als Prädiktoren für "gutes Sterben" haben.

- Auch die Wirksamkeit von Maßnahmen, die „gutes Sterben“ ermöglichen sollen, muss mit Blick auf patientenorientierte Outcomes - aus der Perspektive von Patienten, Angehörigen sowie aus der Public Health-Perspektive - untersucht werden [3, 36]. Bis jetzt gibt es keine evidenzbasierten und validierten Methoden für ein optimales Timing und die Auswahl von palliativmedizinischen Maßnahmen [28].

- Es gibt auch keine einheitliche Definition der Patienten in der Palliativversorgung und es besteht kein Konsens hinsichtlich der Attribute einer Erkrankung, die eine palliativmedizinische Versorgung notwendig machen [38].

- Neben der breiten Dissemination der entwickelten Konzepte wie Lebensqualität am Ende des Lebens, Qualität des Sterbens und Versorgungsqualität am Ende des Lebens wäre eine Vertiefung palliativmedizinischer Aspekte in der Ausbildung in den Heilberufen und damit eine bessere Vorbereitung der beteiligten Berufsgruppen auf das Thema Sterben wünschenswert [3, 22].

\section{Literatur}

1 Anquinet L, Rietjens JAC, Seale C et al. The practice of continuous deep sedation until death in Flanders (Belgium), The Netherlands, and the U.K.: a comparative study. J Pain Symptom Manage 2012; 44: 33-43

2 Bakitas M, Doyle Lyons K, Hegel MT et al. Effects of a palliative care intervention on clinical outcomes in patients with advanced cancer. The project ENABLE II randomized controlled trial. JAMA 2009; 302: 741-749

3 Borasio GD. Über das Sterben. München: Verlag C. H. Beck; 2012

4 Cohen J, van Landeghem P, Carpentier $\mathrm{N}$ et al. Different trends in euthanasia acceptance across Europe. A study of 13 western and 10 central and eastern European countriesm, 1981-2008. Eur J Public Health 2013; 23: 378-380

5 Cox K, Bird L, Arthur A et al. Public attitudes to death and dying in the UK: a review of published literature. BMJ Support Palliat Care 2013; 3: 37-45

6 Dahl E. Terminale Sedierung: Die „Wunderwaffe“ für ein humanes Sterben? Humanes Leben Humanes Sterben 2006; 3: 21-23. Im Internet: http://www.dghs.de/pdf/21_23_HLS3_06.pdf; Stand: 14.8.2015

7 Daveson BA, Alonso JP, Calanzani $\mathrm{N}$ et al. Learning from the public: citizens describe the need to improve end-of-life care access, provision and recognition across Europe. Eur ] Public Health 2014; 24: 521-527
8 Deutsche Hospiz Stiftung. Meinungen zum Sterben - Emnid-Umfrage 2001. Dortmund: Deutsche Hospiz Stiftung, 2001

9 Deutscher Hospiz- und PalliativVerband e. V. Sterben in Deutschland - Wissen und Einstellungen zum Sterben. Berlin: Deutscher Hospiz- und PalliativVerband e. V., 2012

10 Emanuel E], Fairclough DL, Emanuel LL. Attitudes and desires related to euthanasia and physicianassisted suicide among terminally ill patients and their caregivers. JAMA 2000; 284: 2460-2468

11 Ferrand E, Jabre P, Vincent-Genod C et al. Circumstances of death in hospitalized patients and nurses' perceptions: French multicenter Mort-a-l'Hôpital survey. Arch Intern Med 2008; 168: 867-875

12 Geo Wissen. Vom guten Umgang mit dem Tod. Hamburg: Gruner + Jahr, Nr. 51, 2013

13 Göckenjan G. Sterben in unserer Gesellschaft Ideale und Wirklichkeiten. Polit Zeitgesch 2008; 4 : 7-14

14 Granda-Cameron C, Houldin A. Concept Analysis of Good Death in Terminally III Patients. Am J Hosp Palliat Care 2012; 29: 632-639

15 Hales S, Zimmermann C, Rodin G. The Quality of Dying and Death. Arch Intern Med 2008; 168: 912-918

16 Hartmann J, Förstl H, Kurz A. Suizid bei beginnender Dememz - Medizinische und ethische Fragen. Z Med Ethik 2009; 55: 343-350

17 Lorenz KA, Lynn J, Dy SM et al. Evidence for improving palliative care at the end of life: a systematic review. Ann Intern Med 2008; 148 : 147-159

18 Maio G. Gutes Sterben erfordert mehr als die Respektierung der Autonomie. Dtsch Z Onkol 2011; 41: 129-132

19 Mak JMH, Clinton M. Promoting a good death: an agenda for outcomes research - a review of the literature. Nurs Ethics 1999; 6: 97-106

20 Miccinesi G, Rietjens JA, Deliens L, et al. Continuous Deep Sedation: Physicians' Experiences in Six European Countries. J Pain Symptom Manage 2006; 31: 122-129

21 Miyashita M, Sanjo M, Morita T et al. Good death in cancer care: a nationwide quantitative study. Ann Oncol 2007; 18: 1090-1097

22 Morell EA. Learning that a death can be a good death. J Palliat Med 2012; 15: 248-249

23 Müller-Busch HC, Oduncu FS, Woskanjan S et al. Attitudes on euthanasia, physician-assisted suicide and terminal sedation-a survey of the members of the German Association for Palliative Medicine. Med Health Care Philos 2004; 7: 333-339

24 Nelson JE, Puntillo KA, Pronovost PJ et al. In their own words: patients and families define highquality palliative care in the intensive care unit. Crit Care Med 2010; 38: 808-818

25 Patrick DL, Curtis JA, Engelberg RA et al. Measuring and improving the quality of death and dying. Ann Int Med 2003; 139: 410-415

26 Pierson CM, Curtis JR, Patrick DL. A good death: a qualitative study of patients with advanced AIDS. AIDS Care 2002; 14: 587-598

27 Pinzon L, Münster E, Fischbeck S et al. End-of-lifecare in germany: study design, methods and first results of the EPACS study. BMC Palliat Care 2010; 9: 16

28 Qaseem A, Snow V, Shekelle P et al. Evidencebased interventions to improve palliative care of pain, dyspnea, and depression at the end of life: a clinical practice guideline from the American College of Physicians. Ann Int Med 2008; 148: 141-146

Vollständiges Literaturverzeichnis unter http://dx.doi.org/10.1055/s-0041-102676

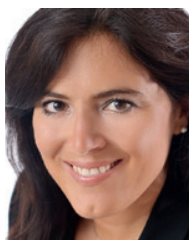

Dr. Claudia Bozzaro ist Medizinethikerin und Philosophin am Institut für Ethik und Geschichte der Medizin der Universität Freiburg

bozzaro@egm.uni-freiburg. de

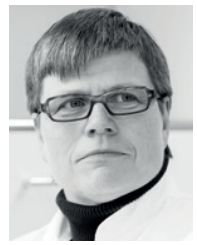

Prof. Dr. Gerhild Becker ist Ärztliche Direktorin der Klinik für Palliativmedizin des Universitätsklinikums Freiburg gerhild.becker@uniklinikfreiburg.de

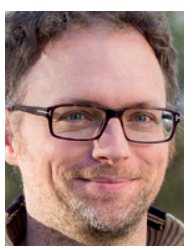

PD Dr. Harald Baumeister ist kommissarischer Leiter der Medizinischen Psychologie und Medizinischen Soziologie sowie Forschungsgruppenleiter in der Abteilung für Rehabilitationspsychologie und Psychotherapie der Universität Freiburg baumeister@psychologie. uni-freiburg.de

Interessenkonflikt

Die Autoren geben an, dass kein Interessenkonflikt besteht.

DOI 10.1055/s-0041-102676

Dtsch Med Wochenschr 2015; 140: 1296-1301 (c) Georg Thieme Verlag KG . Stuttgart · New York . ISSN 0012-0472 
29 Radbruch L, Pyne S. Standards und Richtlinien für Hospiz- und Palliativversorgung in Europa: Teil 1. Weißbuch zu Empfehlungen der Europäischen Gesellschaft für Palliative Care (EAPC).

Z Palliativmedizin 2011; 12: 216-227

30 Rietjens ], Van Der Heide A, Onwuteaka-Philipsen $\mathrm{BD}$ et al. Preferences of the Dutch general public for a good death and associations with attitudes toward end of life decision-making. Palliat Med 2006; 20: 685-692

31 Sepúlveda C, Marlin A, Yoshida T et al. Palliative Care: The World Health Organization's Global Perspective. J Pain Symptom Manage 2002; 24: 91-96

32 Statistisches Bundesamt. Gesundheit - Todesursachen in Deutschland. Wiesbaden: Statistisches Bundesamt, 2011

33 Steinhauser KE, Christakis NA, Clipp EC et al. Factors considered important at the end of life by patients, family, physicians, and other care providers. JAMA 2000; 284: 2476-2482

34 Steinhauser KE, Clipp EC, McNeilly M et al. In search of a good death: observations of patients, families, and providers. Ann Int Med 2000; 132: 825-832
35 Stewart AL, Teno J, Patrick DL et al. The concept of quality of life of dying persons in the context of health care. J Pain Symptom Manage 1999; 17: 93-108

36 Stößel U. Die gesellschaftliche Sicht auf das Sterben. Public Health Forum 21; 80: 10-11

37 SUPPORT Principal Investigators. A controlled trial to improve care for seriously ill hospitalized patients: the study to understand prognoses and preferences for outcomes and risks of treatments (SUPPORT). JAMA 1995; 274: 1591-1598

38 Van Mechelen W, Aertgeerts B, De Ceular K et al. Defining the palliative care patient: a systematic review. Palliat Med 2013; 27: 197-208

39 Von Bredow R, Bruhns A, Dworschak M et al. Zu blau der Himmel. Spiegel 2012; 22: 110-120

40 Walters $G$. Is there such a thing as a good death? Palliat Med 2004; 18: 404-408 